\title{
Acid-Sensing Ion Channels in Blood Volume Regulation
}

\author{
Mitsuhiko Yamada, MD, PhD
}

$\mathbf{P}$ recise regulation of the volume and osmolarity of body fluids is fundamental to survival. A change in blood volume is sensed by cardiopulmonary baroreceptors (low-pressure receptors) that are located in the junctions between the right atrium and venae cavae and between the left atrium and pulmonary veins (Figure). Mechanical stretch of these baroreceptors due to blood volume expansion (BVE) quickly causes diuresis, natriuresis and kaliuresis. Atrial natriuretic peptides (ANPs) secreted by atrial cardiac myocytes play a pivotal role in this response. ${ }^{1}$ An initial study showed that atrial distension in an isolated rat heart-lung preparation induced ANP secretion and it was suggested that cardiac innervation was not essential for ANP secretion; ${ }^{2}$ however, subsequent studies showed that the central nervous system, especially the anteroventral portion of the third ventricle (AV3V), plays an important role in atrial secretion of ANP in response to BVE. ${ }^{1,3,4}$ Atrial stretch due to BVE is sensed by low-pressure baroreceptors altering their afferent input to the nucleus tractus solitaries. This signal is relayed by the locus ceruleus, dorsal raphé nucleus and several interneurons in the AV3V and causes the secretion of oxytocin and other hormones from the neurohypophysis. Oxytocin in circulating blood in turn induces ANP secretion through oxytocin receptors in atrial cardiac myocytes. However, the molecular mechanism by which low-pressure baroreceptors sense atrial distension has not been identified.

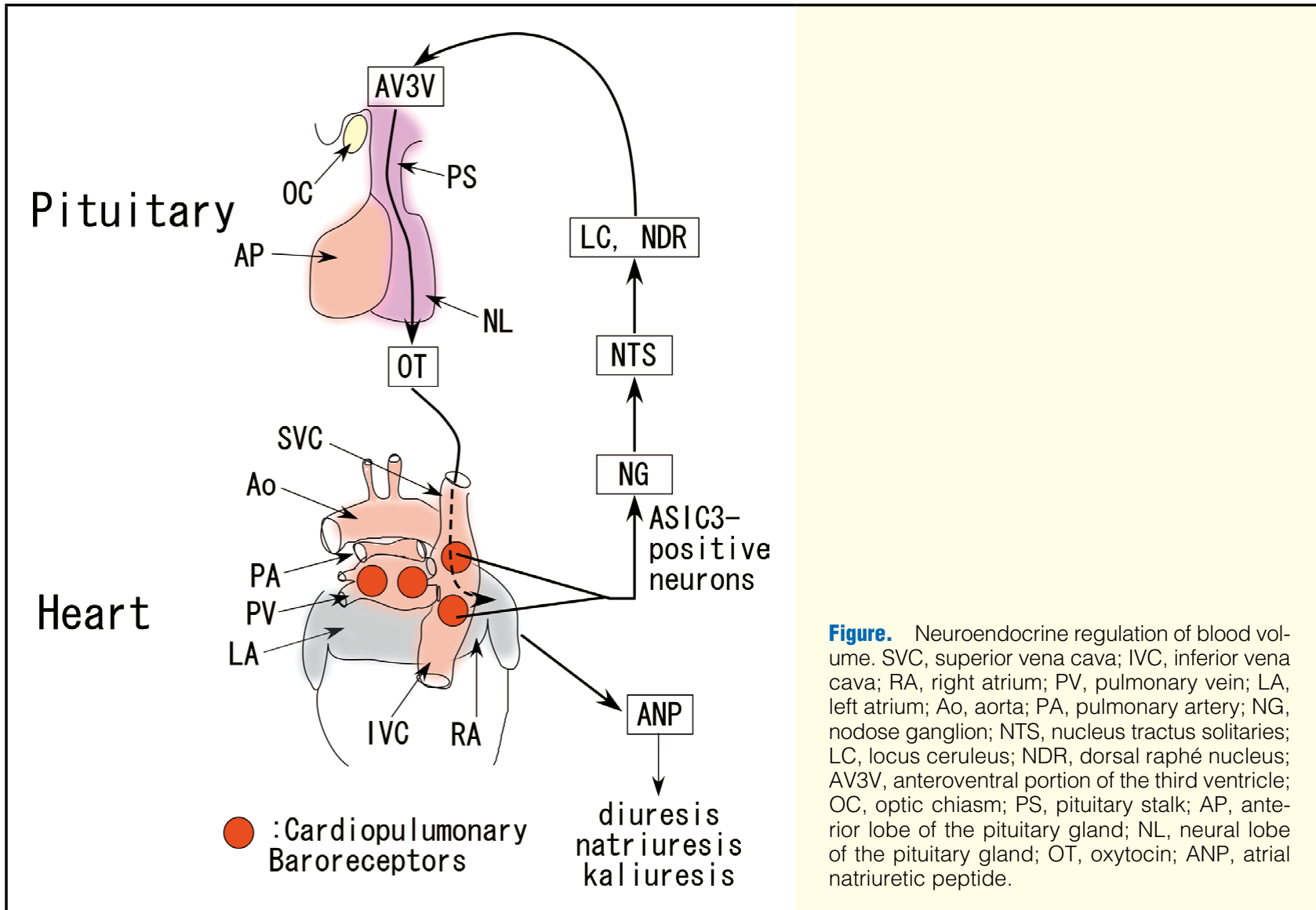

The opinions expressed in this article are not necessarily those of the editors or of the Japanese Circulation Society.

Received January 23, 2011; accepted January 24, 2011; released online February 4, 2011

Department of Molecular Pharmacology, Shinshu University School of Medicine, Matsumoto, Japan

Mailing address: Mitsuhiko Yamada, MD, PhD, Department of Molecular Pharmacology, Shinshu University School of Medicine, 3-1-1

Asahi, Matsumoto 390-8621, Japan. E-mail: myamada@shinshu-u.ac.jp

ISSN-1346-9843 doi:10.1253/circj.CJ-11-0085

All rights are reserved to the Japanese Circulation Society. For permissions, please e-mail: cj@j-circ.or.jp 


\section{Article p 874}

In this issue of the Journal, Lee et al report that acid-sensing ion channels (ASICs) 3 in sensory neurons innervating the venoatrial junction area mediate atrial ANP secretion and diuresis in response to BVE. ${ }^{5}$ ASICs are voltage-insensitive cation channels activated by extracellular protons and blocked by amiloride, ${ }^{6,7}$ and belong to the ENaC/DEG (epithelial amiloride-sensitive $\mathrm{Na}^{+}$channel and degenerin) family of ion channels. An ASIC subunit possesses intracellular $\mathrm{N}$ - and $\mathrm{C}$-termini and 2 hydrophobic transmembrane regions separated by a large extracellular domain. Functional ASICs probably assemble as homo- and heterotetramers. The ASIC family in mammals comprises 4 different genes encoding 7 isomers (ASIC1a, 1b, 2a, 2b, 3 and 4). ASIC channels mainly conduct $\mathrm{Na}^{+}$although homomeric ASIC1a channels are permeable to $\mathrm{Ca}^{2+}$. At the physiological membrane potential, protons trigger a transient inward ASIC current that desensitizes rapidly. An inward ASIC current is capable of inducing action potentials in neurons. Almost all ASIC isoforms are present in primary sensory neurons of the trigeminal, vagal and dorsal root ganglia innervating certain somatic and visceral receptors. ASICs have been thought to be involved in the perception of pain in conditions associated with tissue acidosis. In cardiac afferents, ASIC 3 has been proposed to be the sensor of myocardial acidity that triggers angina during cardiac ischemia. ${ }^{8}$

Several members of the ENaC/DEG family of ion channels have also been proposed to participate in mechanosensation. ${ }^{6,7}$ ASIC2a and ASIC3 are expressed in mechanoreceptors such as Meissner corpuscles; however, their contribution to cutaneous sensation is rather moderate. ASIC1, ASIC2, and ASIC3 also contribute to visceral mechanosensation. ASIC2 is expressed in aortic baroreceptor neurons in the nodose ganglion and their nerve terminals, and ASIC2 null mice have an impaired high-pressure baroreceptor reflex. ${ }^{9}$ ASIC 3 knockout mice have markedly decreased mechanosensitivity in almost all classes of visceral afferents. ${ }^{10}$ At present, how mechanical stress activates ASIC channels is unknown.

Lee et al. showed that Asic3 null (Asic $\left.3^{-1-}\right)$ mice exhibited impaired diuresis in response to BVE compared with wildtype littermates $\left(\right.$ Asic $\left.3^{+/+}\right) .{ }^{5} \mathrm{GdCl}_{3}$, a non-selective blocker of stretch-activated channels, also reduced diuresis in response to BVE in both $\mathrm{Asic} 3^{+/+}$and Asic $3^{-/-}$mice. Statistical analysis showed no interaction between genotypic and $\mathrm{GdCl}_{3}$ effects, indicating that ASIC3 may mediate $\mathrm{GdCl}_{3}$-insensitive diuresis in response to BVE. The authors found that the nodose ganglion and dorsal root ganglia at C8 and T2 levels had neurons responding to BVE in an ASIC3-dependent manner; however, only the nodose ganglion possessed neurons responding to BVE in an ASIC3-dependent but $\mathrm{GdCl}_{3}$-independent manner. They further showed that an increase in plasma ANP concentration in response to BVE was modified by Asic 3 gene disruption and $\mathrm{GdCl}_{3}$ in a manner similar to the response of nodose neurons to BVE. These results indicate that nodose neurons may sense BVE with ASIC3 channels and induce atrial ANP secretion through the central nervous system to cause diuresis.

This study for the first time shows a molecular mechanism by which the nervous system detects BVE in the low-pressure baroreceptor; however, some questions remain unanswered. Although Asic3 gene disruption significantly reduced BVEinduced diuresis, there is a substantial ASIC3-independent fraction of diuresis for which the molecular mechanisms are unknown. This fraction may be mediated by ASIC 2 or other stretch-activated channels responsible for the high-pressure baroreflex and/or renal regulation. ${ }^{9}$ It is also not necessarily clear how much ASIC3 channels participate in $\mathrm{GdCl}_{3}$-sensitive diuresis in response to BVE. The authors found neurons responding to $\mathrm{BVE}$ in an $\mathrm{ASIC} 3$ - and $\mathrm{GdCl}_{3}$-dependent manner in the 3 ganglia, but the functional significance of these neurons was not analyzed. They also found ASIC3 channels in the soma and neurites of these neurons, but the functional role of ASIC3 in the soma remains to be elucidated.

Nevertheless, this study opens up the possibility that ASIC3 channels may be involved in the dysfunction of the lowpressure baroreceptors that occurs in heart failure. It is well known that the inhibitory input from cardiopulmonary as well as atrial baroreceptors to the sympathetic nervous system is decreased in heart failure and that the resultant increase in sympathetic activity causes fatal ventricular arrhythmias and cardiac remodeling. This study suggests that analysis of ASIC3 channels may elucidate the mechanism underlying such dysfunction and that drugs activating ASIC3 may ameliorate the dysfunction of cardiopulmonary baroreceptors in heart failure.

\section{References}

1. Antunes-Rodrigues J, de Castro M, Elias LL, Valenca MM, McCann SM. Neuroendocrine control of body fluid metabolism. Physiol Rev 2004; 84: 169-208.

2. Dietz JR. Release of natriuretic factor from rat heart-lung preparation by atrial distension. Am J Physiol 1984; 247: R1093-R1096.

3. Eskay R, Zukowska-Grojec Z, Haass M, Dave JR, Zamir N. Circulating atrial natriuretic peptides in conscious rats: Regulation of release by multiple factors. Science 1986; 232: 636-639.

4. Antunes-Rodrigues J, Ramalho MJ, Reis LC, Menani JV, Turrin MQ, Gutkowska J, et al. Lesions of the hypothalamus and pituitary inhibit volume-expansion-induced release of atrial natriuretic peptide. Proc Natl Acad Sci USA 1991; 88: 2956-2960.

5. Lee $\mathrm{CH}$, Sun SH, Lin SH, Chen CC. Role of the acid-sensing ion channel 3 in blood volume control. Circ J 2011; 75: 874-883.

6. Kellenberger S, Schild L. Epithelial sodium channel/degenerin family of ion channels: A variety of functions for a shared structure. Physiol Rev 2002; 82: 735-767.

7. Lingueglia E. Acid-sensing ion channels in sensory perception. J Biol Chem 2007; 282: 17325-17329.

8. Sutherland SP, Benson CJ, Adelman JP, McCleskey EW. Acid-sensing ion channel 3 matches the acid-gated current in cardiac ischemiasensing neurons. Proc Natl Acad Sci USA 2001; 98: 711-716.

9. Lu Y, Ma X, Sabharwal R, Snitsarev V, Morgan D, Rahmouni K, et al. The ion channel ASIC2 is required for baroreceptor and autonomic control of the circulation. Neuron 2009; 64: 885-897.

10. Page AJ, Brierley SM, Martin CM, Price MP, Symonds E, Butler R, et al. Different contributions of ASIC channels 1a, 2, and 3 in gastrointestinal mechanosensory function. Gut 2005; 54: 1408-1415. 